\title{
Simultaneous imaging of light and heavy elements at atomic resolution using electron ptychography and fast pixelated detectors
}

Hao Yang ${ }^{1}$, Roberto dos Reis ${ }^{1}$, Colin Ophus ${ }^{1}$, Peter Ercius ${ }^{1}$, Gerardo T. Martinez ${ }^{2}$, Lewys Jones ${ }^{2}$, Martin Huth $^{3}$, Martin Simson ${ }^{3}$, Heike Soltau ${ }^{3}$, Yukihito Kondo ${ }^{4}$, Ryusuke Sagawa ${ }^{4}$, Timothy J. Pennycook ${ }^{5}$, Peter D. Nellist ${ }^{2}$

${ }^{1}$ Molecular Foundry, Lawrence Berkeley National Laboratory, Berkeley, CA, 94720, USA

${ }^{2}$ University of Oxford, Department of Materials, Parks Rd, Oxford, UK

${ }^{3}$ PNDetector GmbH, Sckellstraße 3, 81667 München, Germany

${ }^{4}$ JEOL Ltd.,3-1-2 Musashino Akishima Tokyo 196-8558 Japan

${ }^{5}$ Faculty of Physics, University of Vienna, Vienna, Austria

In the scanning transmission electron microscope (STEM), the commonly-used imaging modes, for example annular dark-field (ADF) or annular bright-field (ABF), form images by using detectors that integrate only a portion of diffraction intensities in the detector plane. Although such integrating detectors used for $\mathrm{ADF}$ and $\mathrm{ABF}$ are able to respond at the timescales required for typical STEM scanning speeds, much of the wealth of information that is present as fine details in the electron diffraction intensity is unavoidably lost. Recent development in detector technology have resulted in cameras with frame-speeds that can exceed $1 \mathrm{kHz}$, and can therefore be used to record a so-called "four dimensional" (4D) dataset, which consists of a 2D diffraction pattem for each probe position of a 2D STEM scan, without inordinate increase in dwell time. Here we explore how the rich diffraction information in the recorded 4D data-set can be extracted efficiently to extend the capabilities of STEM to unlock new applications.

It was shown nearly 20 years ago that ptychography could be used to extract phase information from diffraction pattems that exceeded the conventional diffraction limit of the microscope used [1]. Here we make use of ptychography to form a quantitative image of the phase shift of the electron wave due to the sample, and to use those alongside the simultaneously formed ADF image to characterize the sample. We compare the signal to noise ratio of ptychographic phase as a function of electron dose with a range of other phase imaging approaches, including differential phase contrast STEM and conventional HRTEM approaches $[2,3]$.

We have applied the ptychography approach to a range of materials types. The combination of simultaneous phase imaging and Z-contrast is used to solve the previously unknown structure of a complex nanostructure [4]. We show that residual lens aberrations can be detected and corrected post-acquisition, and that $3 \mathrm{D}$ information can be reconstructed from a single tilt. We also show various examples of imaging light elements in solid state materials [5], and examine the robustness of the ptychography approach to dynamical electron diffraction. [6]

[1] P.D. Nellist, B.C. McCallum, J.M. Rodenburg, Nature, 374 (1995) 630-632.

[2] T.J. Pennycook et al., Ultramicroscopy, 151 (2015) 160-167.

[3] H. Yang, T.J. Pennycook, P.D. Nellist, Ultramicroscopy, 151 (2015) 232-239.

[4] H. Yang et al, Nature Communications 7, (2016) 12532

[5] H. Yang et al, Ultramicroscopy, in press

[6] The authors acknowledge funding from the EPSRC (grant numbers EP/K032518/1 and EP/K040375/1) and the EU Seventh Framework Programme: ESTEEM2. Work at the Molecular Foundry was supported by the Office of Science, Office of Basic Energy Sciences, of the U.S. Department of Energy under Contract No. DE-AC02-05CH11231. 